\title{
Study of a novel method to assist in early reporting of sepsis from the microbiology laboratory
}

\author{
Purabi Barman, Sharmila Sengupta, Shefali Singh \\ Super Religare Laboratories Ltd., Fortis Flt Lt Rajan Dhall Hospital, New Delhi, India
}

\begin{abstract}
Introduction: Microbiology laboratories must provide accurate blood culture reports with rapid turnaround time (TAT) to effectively manage patients with sepsis. In this study three methods are compared for reporting blood culture results: a manual method that included use of a serum separator tube (SST), the conventional manual, and an automated method for identification and susceptibility (ID/AST).

Methodology: Broth from positive blood culture bottles was added to an SST and then centrifuged. The pellet obtained was used to directly inoculate biochemical tests for identification and agar plates for AST on the first day of positivity. Biochemicals and AST plates were read the next day and final results reported on the second day at 24 hours. For conventional disk diffusion testing, the newly positive blood culture broth was also inoculated on solid media on the first day and incubated overnight. The next day AST by was performed as well as biochemical tests from pure colonies. These colonies were also used to inoculate panels for ID/AST using the automated MicroScan 40SI System. These results were recorded on the third day and results reported at 48 hours.

Results: The study included 851 samples Out of 106 (12.4\%) positive blood cultures, 102 were included in the study; Comparison of the 3 methods showed good correlation. Identification was correctly reported in 95 (93.1\%) isolates. The overall AST error rate was 3.8\%,

Conclusions: The use of SST and direct from pellet inoculation reduced TAT for identification and AST results between 18 and 24 hours.
\end{abstract}

J Infect Dev Ctries 2010; 4(12):822-827.

(Received 24 February 2010 - Accepted 13 August 2010)

Copyright ( 92010 Barman et al. This is an open-access article distributed under the Creative Commons Attribution License, which permits unrestricted use, distribution, and reproduction in any medium, provided the original work is properly cited.

\section{Introduction}

Sepsis imposes a significant burden on our health-care system and it is the tenth leading cause of death in the United States [1]. Rapid etiological diagnosis with prompt and adequate antimicrobial therapy is crucial for the successful management of sepsis. Routine laboratory diagnosis relies on automated blood culture methods with continuous monitoring for growth. However, the time to determine blood culture positivity and the growth on subcultured media can take longer than 48 hours to identify and perform antimicrobial susceptibility tests (ID/AST) on the pathogen. Both culture-dependent and independent methods are available for microbial ID/AST. Growth dependent automated systems are valued for their capability of performing both ID and AST from isolated colonies within 24 hours. Rapid, culture-independent methods include molecular tests (PCR) [2] but these require sophisticated equipment, which is too expensive for most diagnostic laboratories in developing countries.
The purpose of the current study was to validate the serum separator tube (SST) method to obtain faster results from blood cultures without incurring any major additional cost. This study compared the ID/AST results, TATs and cost per test of the following three different methods:

i) Conventional manual method of ID and AST (Kirby Bauer disk diffusion) from isolated bacterial colonies.

ii) Automated method for ID/AST using MicroScan 40 SI (Siemens, Frimley, Camberley, UK) from isolated bacterial colonies.

iii) Manual ID and disk diffusion method with the SST directly from pelleted positive blood culture broths.

The aim of the study was to determine if the addition of SST to the routine blood culture methods would be a cost-effective way to reduce the turnaround time (TAT) for final results. 


\section{Methodology}

The study was conducted in the laboratory of a tertiary care hospital in Delhi (national capital region), India, over a period of three months. Blood samples from admitted patients with clinical diagnosis of septicaemia were inoculated in Bactec Plus Aerobic bottles (Becton Dickinson, Sparks, Maryland, USA). These bottles were incubated in the Bactec 9050 instrument (Becton Dickinson, Sparks, Maryland, USA). Once blood culture bottles signalled positive for growth, they were removed from the instrument and Gram stains were prepared to select only samples with a single microscopic morphology for this study. The samples were processed using the following methods: rapid serum separator tube (SST) method, conventional manual and automated methods

\section{Rapid serum separator tube (SST) method}

Broth $(5 \mathrm{~mL})$ from a newly positive blood culture bottle was mixed well, transferred to a SST (BD Vacutainer SST II Advance, Becton Dickinson Sparks, Maryland, USA) and centrifuged (2000g, 10 minutes) to separate bacterial cells from the broth by centrifugation. The supernatant was decanted and the pellet was suspended in Phoenix ID broth (Becton Dickinson, Sparks, Maryland, USA) and adjusted to the 0.5 McFarland turbidity standard using the Phoenix Nephelometer (Becton Dickinson, Sparks, Maryland, USA). This sediment was used to inoculate biochemical tests [3] for bacterial identification and used to perform AST by the disk diffusion method. The biochemical tests included sugar fermentation, indole, methyl red, VogesProskauer, citrate, phenylalanine deaminase, urease, triple sugar iron, amino acid decarboxylase and arginine dihydrolase in addition to oxidase, antisera typing and motility for identification of Gramnegative bacteria. Optochin susceptibility, catalase and coagulase tests were performed for Grampositive bacteria. These ID and AST plates were incubated at $37^{\circ} \mathrm{C}$ overnight. In addition, the suspension was subcultured to $5 \%$ sheep blood agar (BA), chocolate agar (CA) and nutrient agar (NA), which were prepared in-house. ID/AST results were available on the second day and ID/AST results were reported at 24 hours.

\section{Conventional manual and automated methods}

Conventional manual and automated ID/AST methods were considered the gold standard to which the SST method was compared. With the conventional systems, newly positive Bactec bottles were subcultured on BA, CA, Mac-Conkey agar and $\mathrm{NA}$ and incubated at $37^{\circ} \mathrm{C}$ overnight. The next day isolated colonies from solid media were used as inoculum to perform the following tests:

1. Standardized biochemicals (as used in the SST method) and AST by Kirby Bauer disk diffusion method $[3,4]$ were used for ID.

2. ID/AST panels were inoculated with colonies mixed with $25 \mathrm{~mL}$ of inoculum water and adjusted with the MicroScan Turbidity Meter (Siemens, Frimley, Camberley, UK) to reach a final turbidity of the $0.5 \mathrm{McFarland}$ standard. This suspension was then inoculated into the panels by the Renok system Inoculator-D (Siemens, Frimley, Camberley, UK). The MicroScan ID/AST (Siemens, Frimley, Camberley, UK)panels contained some wells with biochemicals, chromogenic substrates, and $\mathrm{pH}$ changes for bacterial identification and other wells were reserved for concentrations of antimicrobial drugs to provide minimum inhibitory concentration (MIC) breakpoint values that could be interpreted as susceptible, intermediate or resistant. The ID/AST breakpoint panels inoculated for Gram-negative bacteria were type NBPC 34 (Siemens, Frimley Camberley, UK) and those for Gram-positive bacteria were type 23. The MICroSTREP plus (Siemens, Frimley, Camberley, UK) panel was used for identification of Streptococci. All panels were placed in the automated MicroScan Walkaway 40 SI System (Siemens, Frimley, Camberley, UK) for results. In both manual and automated conventional methods, reporting was completed in 48 hours since an additional 18 to 24 hours was required for bacterial growth on media plates that had been subcultured from blood broths.

\section{Comparison of SST to the conventional methods}

Bacterial identification results with the SST method were grouped in three different categories: (i) correct identification to the species level; (ii) misidentification at either the genus or species level; and (iii) no identification provided [5].

The AST results by SST method were compared with those from the two conventional methods and data was categorized as very major errors (false susceptibility), major errors (false resistance) and minor errors (resistant to intermediate, intermediate to susceptible, susceptible to intermediate or intermediate to resistant) [6] .The conventional 
Table 1. Antibiotic susceptibility (\%) of bacteria isolated from blood cultures

\begin{tabular}{|c|c|c|c|c|c|c|c|c|c|c|c|c|}
\hline Antibiotics & 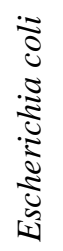 & 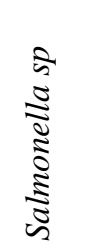 & $\begin{array}{l}\text { के } \\
0 \\
\vdots \\
\vdots \\
0 \\
\vdots \\
0 \\
0 \\
2\end{array}$ & 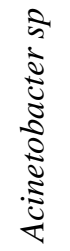 & 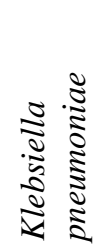 & 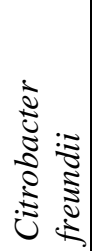 & 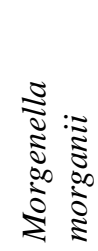 & 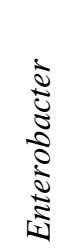 & 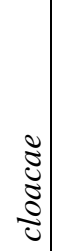 & 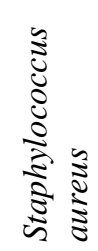 & 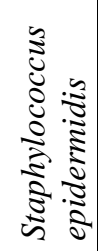 & 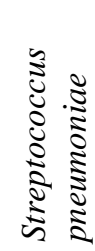 \\
\hline Ampicillin & 0 & 92.8 & NT & NT & 0 & 0 & 0 & 0 & & 5.7 & 50 & NT \\
\hline Amikacin & 85 & NT & 46.6 & 0 & 66.6 & 75 & 100 & 100 & & NT & NT & NT \\
\hline Amoxycillin/clavulanic acid & 30 & 92.8 & NT & NT & 33.3 & 50 & 100 & 50 & & 77.1 & 50 & 100 \\
\hline Ampicillin/Salbactum & 15 & 92.8 & NT & 0 & 33.3 & 50 & 100 & 50 & & 77.1 & 50 & NT \\
\hline Aztreonem & 20 & NT & 33.3 & 0 & 33.3 & 50 & 100 & 50 & & NT & NT & NT \\
\hline Cephalothin & 10 & NT & NT & NT & 33.3 & 25 & 0 & 50 & & NT & NT & NT \\
\hline Cefazoline & 15 & NT & NT & NT & 33.3 & 25 & 0 & 50 & & 77.1 & 50 & NT \\
\hline Cefuroxime & 15 & NT & NT & NT & 33.3 & 25 & 0 & 50 & & NT & NT & NT \\
\hline Cefotaxime & 15 & 92.8 & 13.3 & 0 & 33.3 & 50 & 100 & 50 & & 77.1 & 50 & 100 \\
\hline Ceftriaxone & 15 & 92.8 & 13.3 & 0 & 33.3 & 50 & 100 & 50 & & 77.1 & 50 & 100 \\
\hline Ceftazidime & 15 & 92.8 & 33.3 & 0 & 33.3 & 50 & 100 & 50 & & NT & NT & NT \\
\hline Cefepime & 20 & 100 & 40 & 0 & 33.3 & 50 & 100 & 100 & & 77.1 & 50 & 100 \\
\hline Ciprofloxacin & 10 & 85.7 & 40 & 0 & 33.3 & 50 & 100 & 50 & & 37.1 & 50 & NT \\
\hline Ertapenem & 95 & NT & NT & NT & 100 & 100 & 100 & 100 & & NT & NT & NT \\
\hline Gentamicin & 35 & NT & 33.3 & 0 & 33.3 & 75 & 100 & 50 & & 28.5 & 50 & NT \\
\hline Imipenem & 95 & NT & 60 & 20 & 100 & 100 & 100 & 100 & & NT & NT & NT \\
\hline Levofloxacin & 10 & 85.7 & 40 & 0 & 33.3 & 50 & 100 & 50 & & 37.1 & 50 & 100 \\
\hline Moxifloxacin & 10 & 85.7 & 40 & NT & 33.3 & 50 & 100 & 50 & & 80 & 50 & NT \\
\hline Piperacillin/Tazobactum & 75 & 92.8 & 60 & NT & 100 & 100 & 100 & 100 & & NT & NT & NT \\
\hline Meropenem & 95 & NT & 60 & 20 & 100 & 100 & 100 & 100 & & NT & NT & NT \\
\hline Tobramycin & 50 & NT & 46.6 & 0 & 66.6 & 75 & 0 & 50 & & NT & NT & NT \\
\hline Tetracycline & 15 & 92.8 & NT & 20 & 33.3 & 50 & 0 & 0 & & 77.1 & 50 & 100 \\
\hline Trimethoprim/Sulfamethaxole & 15 & 92.8 & NT & NT & 33.3 & 25 & 0 & 0 & & 80 & 100 & 100 \\
\hline Penicillin & NT & NT & NT & NT & NT & NT & NT & NT & & 5.7 & 50 & 100 \\
\hline Oxacillin & NT & NT & NT & NT & NT & NT & NT & NT & & 77.1 & 50 & NT \\
\hline Clindamycin & NT & NT & NT & NT & NT & NT & NT & NT & & 80 & 100 & 100 \\
\hline Linezolide & NT & NT & NT & NT & NT & NT & NT & NT & & 100 & 100 & NT \\
\hline Vancomycin & NT & NT & NT & NT & NT & NT & NT & NT & & 100 & 100 & NT \\
\hline Erythromycin & NT & NT & NT & NT & NT & NT & NT & NT & & 42 & 50 & 100 \\
\hline
\end{tabular}

methods were considered the reference methods and hence were used for patient reporting.

TAT for each test was defined from the time a blood culture bottle signalled positive to the final release of the ID/AST report.

Cost per test by all the three different methods was calculated.

\section{Results}

A total of 851 samples were included in the study. Of these, 106 (12.4\%) blood cultures were positive, $69(65 \%)$ samples were positive within 12 to
24 hours, and $24(22.6 \%)$ were positive between 24 to 48 hours.

Out of 106 positive blood culture samples, 102 were included in the study as two were of mixed morphology on Gram stain and two grew yeast. Sixty-four $(60.3 \%)$ were identified as Gram-negative bacteria, and 38 (35.8\%) were Gram-positive bacteria.

\section{Identification of positive blood cultures from Bactec}

The isolates identified during the study were 
Table 2. Discordant AST results

\begin{tabular}{|c|c|c|c|}
\hline Antibiotics & Conventional methods & SST & Errors \\
\hline & \multicolumn{3}{|l|}{ Pseudomonas aeruginosa } \\
\hline Ciprofloxacin & $36.3 \%$ & $27.2 \%$ & 1 \\
\hline \multirow[t]{3}{*}{ Meropenem } & $63.6 \%$ & $54.5 \%$ & 1 \\
\hline & & & $\begin{array}{l}\text { Minor errors: } 2 \\
(1.9 \%)\end{array}$ \\
\hline & \multicolumn{3}{|l|}{ Staphylococcus aureus } \\
\hline Penicillin & $5.7 \%$ & $2.8 \%$ & 1 \\
\hline \multirow[t]{2}{*}{ Levofloxacin } & $37.1 \%$ & $34.2 \%$ & 1 \\
\hline & & & $\begin{array}{ll}\text { Major errors: } 2 \\
(1.9 \%) & \\
\end{array}$ \\
\hline
\end{tabular}

Escherichia coli 20 (19.6\%), Salmonella typhi 10 (9.8\%), Pseudomonas aeruginosa 11 (10.7\%), Pseudomonas fluorescens 4 (3.9\%), Acinetobacter sp 5 (4.9\%), Salmonella paratyphi A 4 (3.9\%), Klebsiella pneumoniae 3 (2.9\%), Citrobacter freundii 4 (3.9\%), Morganella morganii 1 (0.9\%), Enterobacter cloacae 2 (1.9\%), Staphylococcus aureus 35 (34.3\%), Staphylococcus epidermidis 2 (1.9\%) and Streptococcus pneumoniae 1 (0.9\%). Identification by all three methods was comparable. The SST method correctly identified 95 (93.1\%) of the isolates; however, $6(5.8 \%)$ could not be identified to the species level (4 P. fluorescens and 2 $S$. epidermidis) and one isolate $(<1 \%)$ of $S$. pneumoniae could not be identified.

\section{Antimicrobial susceptibility test}

The disk diffusion AST method was used with both SST and the conventional manual method. The susceptibility testing for the conventional automated method was performed using MicroScan Walkaway 40SI panels. The AST results of all isolates tested by both conventional methods are shown in Table 1.

The correlation of all AST methods showed discordant results with two isolates. One isolate of $P$. aeruginosa was intermediate to ciprofloxacin and meropenem by the SST method and was susceptible by both conventional methods (minor error, 1.9\%). An isolate of $S$. aureus was resistant to penicillin and levofloxacin by the SST method and susceptible by both conventional methods (major error, 1.9\%). These data are summarized in Table 2 .

\section{Turnaround time}

The TAT of the SST method was 24 hours after a blood culture bottle signalled positive by the Bactec instrument, whereas 48 hours were needed when using conventional manual and automated methods.

Cost

The approximate costs per test were $\$ 5.34, \$ 14.46$ and $\$ 5.69$ for the conventional manual method, conventional automated method, and SST, respectively. The cost of the SST method was comparable to the manual conventional method. However, the automated ID/AST method was more than twice the price per test compared to the other methods studied.

\section{Discussion}

Blood cultures are a critical diagnostic test to guide management of patients with sepsis and they are important for instituting prompt and appropriate antimicrobial therapy. The significance of providing rapid and reliable information to clinicians when a blood culture first becomes positive and reduction in TAT to final results has been well documented $[7,8]$. Although conventional blood culture methods are the gold standard, the turnaround time to results for ID/AST is delayed by 18 to 24 hours to permit colonial growth from subcultures of positive blood culture bottles. The SST method of direct inoculation for positive blood broth reduces TAT by 18 to 24 hours; however, the major limitation is the inability to rapidly identify polymicrobial bacteremia.

The ability of the MicroScan Walkaway (Siemens, Frimley, Camberley, UK) to identify isolates to the species level and provide MIC breakpoints to antimicrobial agents makes the reporting system more reliable, standardized, user friendly and less prone to handling error. However, the requirement for subculturing to obtain isolated bacterial colonies increases the TAT by one day. In addition, there are considerable capital and recurring costs inherent in this automated system. Several studies have reported some 
success with ID/AST testing in automated systems directly from newly positive blood culture broths, although this technique is not standardized recommended protocol and requires further study $[9,10,11]$. Similarly, the adaptation of the SST method to automated ID/AST systems also deserves evaluation to determine the accuracy of this approach.

The cost per test was evaluated for all the three methods and clearly, the SST method was comparable to the conventional manual method; however, the automated system was more than twice the price. The intangible costs of rapid blood culture testing cannot easily be assigned a monetary value; however, they include reduced TAT for results, which can lead to improved patient management and to the selection of appropriate antimicrobial agents. Resources for health research are limited in developing countries and should be directed at finding effective and economical test algorithms.

Although conventional ID/AST methods remain the gold standard, the SST modification has the main advantage of providing ID/AST results one day earlier. The major limitation of the protocol includes the exclusion of polymicrobial blood culture. The SST method correctly identified $95(93.1 \%)$ of isolates to species level; however, one isolate of $S$. pneumoniae could not be identified and $6(5.8 \%)$ could not be assigned a species identification. A similar study done by Trenholme et al. also reported discrepancies in identifying the isolates [12].

Few discrepancies were observed in AST results by the SST method. An overall category error rate of $10 \%$ had been reported as acceptable performance, including $1.5 \%$ very major errors and $3 \%$ major errors [5]. The data from the current study resulted in no very major errors, $1.9 \%$ major errors (one isolate of $S$. aureus) and $1.9 \%$ minor errors (one isolate of $P$. aeruginosa). Discrepancies in AST results using the SST method might be a result of low bacterial concentrations in the direct inoculation of blood culture broth. Other factors such as the presence of red blood cells, cellular debris and constituents of blood culture media or technical error may be additional confounding factors [13]. Some studies have reported discrepancies in results when isolates were directly inoculated from blood culture broths $[5,6,12,14,15]$.

Resource-limited countries need inexpensive, quick and accurate methods for the laboratory diagnosis of sepsis. The SST method as described in this study offers one approach for consideration, reporting that it effectively reduced TAT to results by 18 to 24 hours with a negligible increase in price. This method has the potential to improve patient care and decrease hospital cost. Limitations of the study include the lack of data on fastidious organisms and the limited sample size. Standardization and validation with a larger number of positive blood cultures over a longer period of time is necessary to authenticate the value of the SST method.

\section{Acknowledgments}

We thank Becton Dickinson (Gurgoan, India) for providing Serum Separator tubes, Phoenix broth and Phoenix Nephelometer.

\section{References}

1. Melamed A, Sorvillo FJ (2009) The burden of sepsisassociated mortality in the United States from 1999 to 2005: an analysis of multiple-cause-of-death data. Crit Care 13. Available from http:// www.ncbi.nlm.gov/pmc/articles/pmc2688146. Last accesses on 5th September 2010.

2. Daar A S, Thorsteinsdottir1 H, Martin D K, Smith A C, Nast S, Singer PA (2002) Top ten biotechnologies for improving health in developing countries. Nature Genetics 32: 229232.

3. J.G Collee, R.S Miles, B Watt (1996) Test for Identification of Bacteria. In, J.G Collee, A.G fraser, B.P Marmion, A. Simmons (eds) Mackie and McCartney Practical Medical Microbiology, 14th Edition. Churchill Livingston, 131-149.

4. Performance Standards for Antimicrobial Susceptibility Testing: Eighteenth Informational Supplementation. Clinical Laboratory Standard Institute. M100 - S 19, Vol 29, No 3.

5. Cueto MD, Ceballos E, Martinez-Martinez L, Perea EJ, and Pascual A (2004). Use of Positive Blood Cultures for Direct Identification and Susceptibility Testing with the Vitek 2 System. J of Clin Microbiol 42: 3734-3773.

6. Doern GV, Scott DR, Rashed AL, Kenneth SK (1981) Evaluation of a direct blood culture disk diffusion antimicrobial susceptibility test. Antimicrob Agents and Chemother 696-698.

7. Mette S, Mette N, Schonheyder HC(2007) First notification of positive blood cultures and the high accuracy of the Gram Stain report. J Clin Microbiol 45: 1113-1117.

8. Kerremans JJ, Verboom P, Stijnen T, Hakkaart-van LR, Goessens W, Verbrugh HA, Vos MC (2008). Rapid identification and antimicrobial susceptibility testing reduce antibiotic use and accelerate pathogen-directed antibiotic use. J Antimicrob Chemother 61: 428-435.

9. Ling $\mathrm{T}$ K W, Liu ZK, Augustine FB, Chen (2003) Evaluation of the VITEK 2 System for rapid direct identification and susceptibility testing of gram-negative bacilli from positive blood cultures. J Clin Microbiol 41: 4705-4707.

10. Kiehn TE, Capitolo C, Armstrong D (1982) Comparison of direct and standard microtitre broth dilution susceptibility testing of blood culture isolates. J Clin Microbiol 16: 96-98. 
11. Chapin KC and Musgnug MC (2003) Direct susceptibility testing of positive blood cultures by using Sensititre broth microdilution plates. J Clin Microbiol 41: 4751-4754.

12. Trenholme GM., Kaplan RL, Karakusis PH, Stine T, Fuhrer J, Landau W, Levin S (1989) Clinical impact of rapid identification in susceptibility testing of bacterial blood culture isolates. J Clin Microbiol 27: 1342-1345.

13. Wellinghausen N, Pietzcker T, Poppert S, Belak S, Fieser N, Bartel N, Essig A (2007) Evaluation of the Merlin MICRONAUT System for rapid direct susceptibility testing of gram-positive cocci and gram-negative bacilli from positive blood cultures. J Clin Microbiol 45: 789-795.

14. Waites KB, Brookings ES, Moser SA, Zimmer BL (1998) Direct susceptibility testing with positive BacT/Alert blood cultures by using MicroScan overnight and rapid panels. J Clin Microbiol 36: 2052-2056.
15. Diederen BMW, Zieltjens M, Wetten HV, Buiting AGM (2006) Identification and susceptibility testing of Staphylococcus aureus by direct inoculation from positive BACTEC blood culture bottles. Clin Microbiol Infect 12: 84-86.

\section{Corresponding author}

Purabi Barman

Super Religare Laboratories

Fortis Flt Lt Rajan Dhall Hospital

Vasant Kunj, New Delhi, India

Telephone: 91- 9899094295

Fax: Not available

Email: purabi.barman@gmail.com

Conflict of interests: No conflict of interests is declared. 